\title{
ON REDUCTION EXPONENTS OF IDEALS WITH GORENSTEIN FORMRING*
}

\author{
by M. HERRMANN, C. HUNEKE and J. RIBBE
}

(Received 6th January 1994)

\begin{abstract}
This paper studies questions connected with when the Rees algebra of an ideal or the formring of an ideal is Gorenstein. The main results are for ideals of small analytic deviation, and for $m$-primary ideals of a regular local ring $(R, m)$. The general point proved is that the Gorenstein property forces (and is sometimes equivalent to) lowering the reduction number of the ideal by one from the value predicted if one only assumes the Rees algebra or formring is Cohen-Macaulay.
\end{abstract}

1991 Mathematics subject classification: 13 A30.

\section{Introduction}

In this note we discuss under various aspects the Gorenstein property of the formring $G(I)=\bigoplus_{n \geq 0} I^{n} / I^{n+1}$ of ideals $I$ having small analytic deviation in local Noetherian rings $A$. Our premise is that in several situations the Gorensteinness of $G(I)$ is closely related to low reduction exponents of $I$. By way of illustration consider first the ideal $I$ generated by the Pfaffians of a generic skew symmetric matrix or secondly the ideal generated by the maximal minors of a generic $m \times n$-matrix, $m \geq n$; in both cases $G(I)$ is Gorenstein and the reduction exponent $r(I) \leqq 1$ (cf. [12, Theorem 2.2]). We prove in Theorems 2.1 and 2.3 that this situation in particular holds for ideals $I$ of analytic deviation 1 or 2 satisfying some mild additional conditions. These results are shared with Goto and Nakamura as they were independently obtained by them too but with other methods $[3,4]$. Recently N. V. Trung and Z. Tang tried to formulate similar results also for ideals of analytic deviation $\geq 3$ by using quite a different approach.

A third example which illustrates our premise is an ideal $I$ which is a power of the maximal ideal $m$ in a regular local $\operatorname{ring} A$ of $\operatorname{dim} A \geq 2$. Then the Gorensteinness of $G(I)$ implies that $r(I) \leqq d-2$ by [9, (2.4), (2.6), (3.7), and (3.9)]. One of our main results (see Theorem 2.5) shows that this conclusion is true for any $m$-primary ideal in a regular local ring $(A, m)$. It turns out that the proof of this result uses a new Briançon-Skoda theorem, which was conjectured by the second author and recently proved by J. Lipman ([13]). Note that if one just assumes that $G(I)$ is $C M$ for an $m$-primary ideal $I$ in a regular local ring of dimension $d$, then one can conclude from the usual Briançon-

*The first author was partially supported by the LMS and EMS while visiting the University of Sheffield and the University of Edinburgh. The second author was partially supported by the NSF. 
Skoda theorem that $r(I) \leq d-1$. When $G(I)$ is Gorenstein we obtain bounds better by 1 . Moreover the proof requires a better Briançon-Skoda theorem.

On the other hand one cannot expect a corresponding result for an $m$-primary ideal in a non-regular local ring. As a case in point consider the ideal $I:=m$ in the local ring $(A, m)$ of a hypersurface singularity with multiplicity $e=e(A) \geq 2$. Then we have $r(m)=e-1$ by $[6,(26.4)]$, hence $r(m)$ can be any number although $G(m)$ is always Gorenstein.

Finally we relate in Corollary (2.7) the Gorensteinness of $G(I)$ to the Gorenstein property of the Rees ring $R\left(I^{r}\right)$ for some $r \geqq 1$ assuming that $I$ is an $m$-primary ideal $I$ in a regular local ring $A$ with a Cohen-Macaulay formring. (Recall that the Rees algebra $R(J)$ of an ideal $J$ in a ring $R$ is the ring, $R \oplus J \oplus J^{2} \oplus \ldots$ )

\section{Results and comments}

We always assume that $A / m$ is infinite. We denote the height of an ideal $I \subset A$ by ht $(I)$ or $h$ and the analytic spread of $I$ by $\ell(I)$ or $\ell$. The analytic deviation of $I$ is defined by $a d(I)=\ell(I)-\mathrm{ht}(I) \geq 0$. The case when $a d(I)=0$ has been studied a great deal. In this case $I$ is said to be equimultiple. See [6] for more information.

The term " $I$ is generically a complete intersection" means that $v\left(I_{P}\right)=\mathrm{ht}(P)$ for $P \in \operatorname{Min}(A / I)$, where $v()$ is the least number of generators of an ideal in a local ring $A$.

An ideal $J \subset I$ is called a minimal reduction of $I$ if $J$ is minimal with respect to the property $I^{n+1}=J I^{n}$ for some integer $n \geq 0$. The least such $n$ for which $I^{n+1}=J I^{n}$ is denoted by $r_{J}(I)$, the reduction number of $I$ with respect to $J$. The minimum of all such $r_{J}(I)$ is denoted by $r(I)$, called the reduction number of $I$.

For an element $a \in A, a^{*}$ denotes the initial form of $a$ in $G(I)$. In other words, $a^{*}$ is the image of $a$ in $I^{n} / I^{n+1}$ if $a \in I^{n}$ and $a \notin I^{n+1}$. We will often make use of the fact that the initial forms of the elements forming a minimal reduction of $I$ sit in degree 1 in $G(I)$. Moreover, the minimal primes of $I$ and any reduction $J$ of $I$ are the same. Occasionally if it is clear from context, we will drop the dependence on $I$ of $G(I), r(I)$, etc.

Theorem 2.1. Let $I$ be an ideal of $\operatorname{ad}(I)=1$ in a Gorenstein local ring A. Assume that $I$ is generically a complete intersection. Then the following hold:

(i) if $G(I)$ is Gorenstein then $r(I) \leqq 1$;

(ii) if $r(I) \leqq 1$ and $A / I$ is Cohen-Macaulay, then $G(I)$ is Gorenstein and $r(I)=0$.

The following corollary is an immediate consequence of Theorem 2.1 and of [10, Theorem 2.2].

Corollary 2.2. Under the assumptions as in Theorem 2.1 assume that $A / I$ is CohenMacaulay and there exists a minimal reduction $J$ of $I$ such that $r_{J_{Q}}\left(I_{Q}\right) \leqq 1$ for every prime ideal $Q \supset I$ with $\mathrm{ht}(Q / I)=1$. Then $r(I)=0$.

The proof of Theorem 2.1 is based on the explicit calculation of socle elements in $G(I) / S$, where $S$ is a suitable parameter ideal in $G(I)$. We don't quite see that this 
strategy applies also to deviation 2 ideals. Therefore we use homological methods in this case, i.e. we compute the $a$-invariant of $G(I)$,

$$
a(G(I)):=\max \left\{n \in \mathbb{Z} \mid\left[H_{M}^{d}(G(I))\right]_{n} \neq 0\right\},
$$

where $d=\operatorname{dim} A$ and $H_{M}^{d}(G(I))$ is the $d^{\text {th }}$ (graded) local cohomology of $G(I)$ with respect to the unique maximal homogeneous ideal $M$ of $G(I)$. In [3, Proposition 2.4], the $a$ invariant of $G(I)$ was computed for ideals of deviation 1. We describe in the following Theorem 2.3 an analogous formula for deviation 2 ideals.

Theorem 2.3. Let $I$ be a Cohen-Macaulay ideal of $\operatorname{ad}(I)=2$ and $h t(I)=h \geq 2$ in a Gorenstein local ring $A$. Assume that $G(I)$ is Cohen-Macaulay and $I_{P}$ is a complete intersection for all $P \supseteq I$ such that $\mathrm{ht}(P / I) \leqq 1$. Then the following hold:

(i) $a(G(I))=\left\{\begin{array}{lll}r(I)-h-2 & \text { if } & r(I) \geq 2 \\ -h & \text { if } & r(I) \leq 1\end{array}\right.$

(ii) if $G(I)$ is Gorenstein then $r(I) \leqq 1$.

The next proposition is another version of Theorem 9.1 in [7]. It presents a typical example of a deviation 2 ideals with reduction exponent $r(I)=0$; see also [10, Theorem 3.1], and [16, Theorem 10.17], where ideals $I$ generated by weak $d$-sequences are considered.

Proposition 2.4. Let $A$ be a Gorenstein local ring and $I$ an ideal of height $>0$ with $v(I)=\mathrm{ht}(I)+2$. Assume that $A / I$ is Cohen-Macaulay and $v\left(I_{P}\right) \leq \mathrm{ht}(P)$ for every prime $P \supset I$. Then $I$ is an ideal of $\operatorname{ad}(I)=2$ with Gorenstein formring $G(I)$ and reduction exponent $r(I)=0$. Moreover $I$ is generated by a $d$-sequence.

Remark. If we replace the condition $v\left(I_{P}\right) \leq \mathrm{ht}(P)$ for all $P \supset I$ in Proposition 2.4 by the stronger one $v\left(I_{P}\right) \varsubsetneqq \mathrm{ht}(P)$ for all $P \supsetneqq I$ and if we assume that $I_{P}$ is a parameter idedal in $A_{P}$ for all $P \in \operatorname{Min}(A / I)$ then $G(I)$ is $A / I$-torsion free (cf. [7, Theorem 9.2]). Hence if $I=Q$ is a prime ideal, then the ordinary and the symbolic powers of $Q$ coincide: $Q^{n}=Q^{(n)}$ for all $n \geq 1$. Since $v\left(Q_{Q}\right)=\ell\left(Q_{Q}\right)$ in our case, this fact is closely related to Theorem 3.5 in [10]. One has the following example (cf. [12, Theorem 2.2]): Let $X=\left(X_{i j}\right)$ be a generic skew-symmetric 5 by 5 matrix with zeros down the diagonal over a field $k$. Let $I$ be the ideal generated by the corresponding Pfaffians in $k\left[X_{i j}\right]$. Then $I$ is a height 3 Gorenstein ideal of linear type, minimally generated by 5 elements, and in fact $v\left(I_{P}\right) \leqq$ ht $(P)$ for all $P \supset I$ by $[12$, Theorem 1.16].

Theorem 2.5. Let $(A, m)$ be a noetherian local ring of dimension $d$. Let $I$ be an m-primary ideal with minimal reduction $J=\left(x_{1}, \ldots, x_{d}\right)$, and let $x_{i}^{*}$ be the initial form of $x_{i}$ in $G=G(I)$. Assume that $G$ is Cohen-Macaulay. Let

$$
k=\max \left\{j \mid\left(I^{j}: m\right)+J \nsubseteq I^{j}+J\right\} .
$$


Then $k \geq 1$ and $G /\left(x_{1}^{*}, \ldots, x_{d}^{*}\right)$ has socle elements in degree $k-1$. If $G$ is Gorenstein then $k-1=r_{J}(I)$. Moreover, if $d \geq 2$ and $A$ is regular then $k \leq d-1$, so in this case $r_{J}(I) \leqq d-2$.

Corollary 2.6. Adopt the assumptions of Theorem 2.5. Also assume that $A$ is regular and that $G(I)$ is Gorenstein. Then $k=1$ if and only if $A / I$ is Gorenstein.

The ideal $I$ is equimultiple in Theorem 2.5, hence if $r(I) \leq d-1$ then $G(I)$ is Cohen-Macaulay if and only if $R(I)$ is Cohen-Macaulay (see [6]). This last condition is satisfied in the regular ring $A$ by the Briançon-Skoda Theorem ([1]). In the following corollary to Theorem 2.5 we formulate an analogous statement for the Gorenstein property of $G(I)$ and $R\left(I^{q}\right), q \geq 1$; see also Theorem 2.3 in [8].

Corollary 2.7. Let $(A, m)$ be a regular local ring of dimension $\geq 2$ and $I$ an m-primary ideal in A. Assume that $G(I)$ is Cohen-Macaulay. Then the following are equivalent:

(i) $G(I)$ is Gorenstein;

(ii) there exists an integer $q \geqq 1$ such that $R\left(I^{q}\right)$ is Gorenstein.

\section{Proof of Theorems 2.1 and 2.3}

Before we begin the proofs of Theorems 2.1 and 2.3, we state a fundamental lemma of Goto and Huckaba [3, Lemma 2.2] which we will use several times:

Lemma 3.0. Let $R=\bigoplus_{n \geq 0} R_{n}$ be a noetherian graded ring over a local ring $\left(R_{0}, m\right)$. Let $X$ be a finitely generated graded $R$-module with $X_{n}=0$ for all $n \gg 0$. Then for all integers $k, n$ we have an isomorphism $\left[H_{N}^{k}(X)\right]_{n} \cong H_{m}^{k}\left(X_{n}\right)$ of $R_{0}$-modules, where $N=$ $m R+R^{+}$is the maximal homogeneous ideal of $R$.

3.1. Proof of Theorem 2.1. First note that in parts (i) and (ii) of this theorem $G(I)$ is Cohen-Macaulay by [11, Theorem 2.1]. Then Theorem 2.1 (and Corollary 2.2) reduce to the case $\operatorname{dim} A=1, \operatorname{ht}(I)=0, I$ is generically 0 and $\ell(I)=1$ by $[3$, proof of Proposition 2.4].

Before we begin the proof in this case, we note a couple of facts concerning the annihilators of ideals and the primary decomposition of $(0)$. Since $I$ is generically 0 , it follows that for every prime $P$ minimal over $I$, the primary component of $I$ along $P$ is the same as that of $(0)$ along $P$. In particular $(0: I)$ will lie outside all of these minimal primes and is also unmixed, as $A$ is Cohen-Macaulay. It is easy to see from primary decompositions that $I=(0:(0: I))$ iff $I$ is unmixed. Since the dimension of $A$ is 1 , this holds iff $A / I$ is Cohen-Macaulay, which we assume for part (ii), but not in part (i).

Choose an element $x \in I$ such that $J=(x)$ is a minimal reduction of $I$. Choose $c \in(0: I)$ but not in the union of primes $P \supseteq I$ such that ht $(P)=0$. This choice is possible since $I$ is generically 0 . Then $0: c=0: 0: I$ and $x+c$ is a parameter in $A$. Moreover $x^{*}+c^{*}$ is a regular element on $G=G(I)$ and we observe the following (for details see in particular $[1, \S 5])$; 


$$
\sqrt{\left(x^{*}+c^{*}\right)}=\sqrt{\left(x^{*}, c^{*}\right)}=M, \text { the unique maximal homogeneous ideal. }
$$

For an ideal $Q$ in $G(I)$ let $Q_{2 j}$ signify $Q \cap\left(G_{j} \oplus G_{j+1} \oplus \cdots\right)$. Since $c^{*} G_{+}=0$, we have

$$
\left(x^{*}+c^{*}\right)_{\geq 2}=\left(x^{*}\right)_{\geq 2}=\left(x^{*}, c^{*}\right)_{\geq 2}
$$

As $\left(x^{*}+c^{*}\right) \cap G_{+} \subsetneq\left(x^{*}\right)$

$$
\left(x^{*}+c^{*}\right) \varsubsetneqq\left(x^{*}, c^{*}\right) .
$$

If $G$ is Gorenstein then $G /\left(x^{*}+c^{*}\right)$ is an Artinian Gorenstein ring and $\operatorname{soc}\left(G /\left(x^{*}+c^{*}\right)\right)$ is 1-dimensional. Since $x^{*}$ is not in $\left(x^{*}+c^{*}\right)$, an element representing the socle can be chosen to be a multiple of $x^{*}$. We claim that this forces $G_{2} \subseteq\left(x^{*}+c^{*}\right)$. If not, another socle representative $w^{*}$ could be chosen which is homogeneous of degree at least two. But then that element will be in the ideal $\left(x^{*}+c^{*}, x^{*}\right)$ and by (2) this forces $w^{*} \in\left(x^{*}+c^{*}\right)$, which is a contradiction. Hence $G_{2} \subseteq\left(x^{*}+c^{*}\right)$, and by (2), it follows that $r(I) \leq 1$, which proves (i) of Theorem 2.1.

To prove (ii) it is enough to show that

$$
\operatorname{dim}\left[\operatorname{soc}\left(G /\left(x^{*}+c^{*}\right)\right]=1 .\right.
$$

Now since $I \subsetneq(x+c)$ (note that $I:(x+c)=I: c=I)$ and $\operatorname{dim}(\operatorname{soc}(A /(x+c))=1$, we can choose an element $w \in I$ which represents the socle modulo $(x+c) A$. We claim that the initial form $w^{*} \in G_{1}$ (note that $w$ cannot be in $I^{2}$ since $I^{2}=x I \subseteq(x+c)$ ) represents the socle of $G /\left(x^{*}+c^{*}\right)$. To prove this, observe first that $G_{\geq 2} \subseteq\left(x^{*}+c^{*}\right)$, hence any socle element modulo $\left(x^{*}+c^{*}\right)$ can be represented by $a_{0}^{*}+a_{1}^{*}$, where $a_{0}^{*} \in G_{0}, a_{1}^{*} \in G_{1}$ (possibly one is zero). Let $a_{0}^{*}+a_{1}^{*}$ be an arbitrary nonzero socle element modulo $\left(x^{*}+c^{*}\right)$.

Step 1: Since $G_{+}\left(a_{0}^{*}+a_{1}^{*}\right) \equiv 0 \bmod \left(x^{*}+c^{*}\right)$ we get

$$
x^{*}\left(a_{0}^{*}+a_{1}^{*}\right)=\left(x^{*}+c^{*}\right) \cdot\left(b_{0}^{*}+b_{1}^{*}+b_{2}^{*}+\cdots\right) .
$$

Then we must have that $c \cdot b_{0} \in I$, hence $c \cdot b_{0} \in I \cap(0: I)=(0)$ (by definition of $c$ ). Then $b_{0} \in 0: c \subseteq 0: 0: I=I$ (note that in part (ii) we are assuming that $A / I$ is Cohen-Macaulay), i.e. $b_{0}^{*}=0$. This implies $x^{*} \cdot a_{0}^{*}=c^{*} \cdot b_{1}^{*}=0$, since $c^{*} G_{+}=0$. Therefore $x \cdot a_{0} \in I^{2}=x \cdot I$ (note that $r(I) \leq 1)$. Hence

$$
a_{0} \in I+(0: x)=I+(0: I)
$$

(To see that $(0 ; x)=(0: I)$, observe that $(x)$ and $I$ have the same minimal primes as $x$ is a reduction of $I$. Since $I$ and therefore $(x)$ is generically 0 , their annihilators are simply the intersection of the primary components of 0 whose primes do not contain $I$.) Therefore we may assume $a_{0} \in 0: I$ in the sequel.

Step 2: Let $y \in m \backslash I$; since $m \cdot\left(a_{0}^{*}+a_{1}^{*}\right) \equiv 0 \bmod \left(x^{*}+c^{*}\right)$ we get $y^{*}\left(a_{0}^{*}+a_{1}^{*}\right)=$ $\left(x^{*}+c^{*}\right) \cdot\left(b_{0}^{*}+b_{1}^{*}+\cdots\right)$. As $c^{*} b_{1}^{*}=0$ we get the following equations: 


$$
\begin{gathered}
y a_{0}-c b_{0} \in I \\
y a_{1}-x b_{0} \in I^{2}
\end{gathered}
$$

Since $c, a_{0} \in 0: I$, we have $y a_{0}-c b_{0} \in I \cap(0: I)=(0)$, hence $y a_{0}=c b_{0}$. Moreover $y a_{1}-$ $x b_{0} \in I^{2}=x I$. Therefore we can freely change $b_{0}$ by an element of $I$ (noting that $c I=0$, so that the first equation does not change) and assume that

$$
y a_{0}=c b_{0} \text { and } y a_{1}=x b_{0}
$$

Thus $y\left(a_{0}+a_{1}\right)=b_{0}(x+c)$.

Since $y$ is arbitrary in $m \backslash I$ we get finally $m\left(a_{0}+a_{1}\right) \subseteq(x+c)$. So $a_{0}+a_{1}$ is in the socle of $A /(x+c)$, hence

$$
a_{0}+a_{1}=\alpha w+(x+c) \cdot t, \quad \alpha, t \in A
$$

Step 3: If $\alpha \in m$ in (4), then $a_{0}+a_{1}=(x+c) \cdot u, u \in A$; hence

$$
a_{0}-c u=-a_{1}+x u \in I \cap(0: I)=0,
$$

i.e. $a_{0}=c u$ and $a_{1}=x u$. This implies that $a_{0}^{*}+a_{1}^{*}=\left(c^{*}+x^{*}\right) \cdot u^{*}$, which contradicts the fact that $a_{0}^{*}+a_{1}^{*}$ is nonzero modulo $\left(c^{*}+x^{*}\right)$. (Here $u^{*}$ is considered as an element of degree zero in $G$.) Therefore $\alpha$ in (4) is a unit, say w.l.o.g. $\alpha=1$, and

$$
a_{0}+a_{1}=w+(x+c) t
$$

Since $w$ was chosen in $I$, we get as above

$$
a_{0}=c t \text { and } a_{1}=w+x t
$$

i.e. by (5)

$$
a_{0}^{*}+a_{1}^{*}-\left(x^{*}+c^{*}\right)(t+I)=w^{*}
$$

so that $a_{0}^{*}+a_{1}^{*}=w^{*}$ modulo $\left(x^{*}+c^{*}\right)$. Thus $\operatorname{soc}\left(G /\left(x^{*}+c^{*}\right)\right)$ is 1 -dimensional. Since $G$ is Cohen-Macaulay, $G$ must be Gorenstein.

For the proof of $r(I)=0$ we prove in our situation $(h t(I)=0)$ the following formula for the Cohen-Macaulay type of $G(I)$.

Claim: type $(G(I))=v(I)$

For the basic facts on graded local cohomology used in the proof of this claim and for other proofs below, we refer the reader to [6]. We consider the following two exact sequences

$$
0 \rightarrow\left(H_{M}^{1}\left(x^{*} G\right)\right)^{\vee}(-1) \stackrel{x^{*}}{\rightarrow} w_{G} \rightarrow\left(H_{M}^{1}\left(0:_{G} x^{*}\right)\right)^{\vee} \rightarrow 0
$$




$$
0 \rightarrow\left(H_{M}^{1}\left(G / x^{*} G\right)\right)^{\vee} \rightarrow w_{G} \rightarrow\left(H_{M}^{1}\left(x^{*} G\right)\right)^{\vee} \rightarrow\left(H_{M}^{0}\left(G / x^{*} G\right)\right)^{\vee} \rightarrow 0,
$$

where ()$^{\vee}:=\operatorname{Hom}_{G}\left(-, E_{G}\right) \equiv \operatorname{Hom}_{A}\left(-, E_{A}\right)$ denotes the dual with respect to the injective hull $E_{G}$ (resp. $E_{A}$ ) of the residue field $k=G / M=A / m$, and $w_{G}=\operatorname{Hom}_{G}\left(H_{M}^{1}(G), E_{G}\right)$ is the canonical module of $G$ (observe that ( $)^{\vee}$ switches the grading). Note that

$$
\left[0:{ }_{G} x^{*}\right]_{n}=0 \text { for } n \geq 1 \text { and }\left[G / x^{*} G\right]_{n}=0 \text { for } n \geq 2,
$$

since $I^{2}=x I$, and $(0: I) \cap I=0$. Furthermore, $a(G)=0$ by [3, Proposition 2.4]. Since the Cohen-Macaulay-type of $G$ coincides with the minimal number of homogeneous generators of $w_{G}$, it is enough to show that $w_{G}$ is generated by elements of degree zero and that $v\left(\left[w_{G}\right]_{0}\right)=v(I)$. Concerning the first assertion, note that in (6) above we have $\left[\left(H_{M}^{1}\left(\left(0:_{G} x^{*}\right)\right)\right)^{\vee}\right]_{n}=\left(\left[H_{M}^{1}\left(\left(0:_{G} x^{*}\right)\right)\right]_{-n}\right)^{\vee}=\left(H_{m}^{1}\left(\left[\left(0:_{G} x^{*}\right)\right]_{-n}\right)\right)^{\vee}=0$ for all $n \geq 1$ (the first equality holds since the dual $\vee$ switches the grading, and the second equality holds by Lemma 3.0). Furthermore in (7) above we have $\left[\left(H_{M}^{0}\left(G / x^{*} G\right)\right)^{\vee}\right]_{n}=$ $\left(\left[H_{M}^{0}\left(G / x^{*} G\right)\right]_{-n}\right)^{\vee}=0$ for all $n \geq 0$ (that is clear for $n \geq 1$, since $H_{M}^{0}\left(G / x^{*} G\right)$ is a submodule of the positively graded module $G / x^{*} G$; for $n=0$ the argument is as follows:

$$
\left[H_{M}^{0}\left(G / x^{*} G\right)^{\vee}\right]_{0}=\left(\left[H_{M}^{0}\left(G / x^{*} G\right)\right]_{0}\right)^{\vee}=H_{m}^{0}(A / I)^{\vee}=0,
$$

since $A / I$ is 1-dimensional Cohen-Macaulay); now we get, by (6), for $n \geq 1\left[w_{G}\right]_{n}=$ $x^{*}\left[H_{m}^{1}\left(x^{*} G\right)^{\vee}\right]_{n-1}$, where $\left[H_{m}^{1}\left(x^{*} G\right)^{\vee}\right]_{n-1}$ is a homomorphic image of $\left[w_{G}\right]_{n-1}$ by $(7)$; we conclude that $w_{G}=G \cdot\left[w_{G}\right]_{0}$. To show that $v\left(\left[w_{G}\right]_{0}\right)=v(I)$, consider sequence (6) in degree 0 ; note that

$$
\begin{aligned}
{\left[H_{M}^{1}\left(0:_{G} x^{*}\right)^{\vee}\right]_{0} } & =\left(\left[H_{M}^{1}\left(0:_{G} x^{*}\right)\right]_{0}\right)^{\vee} \\
& =\left(H_{m}^{1}\left(\left[0:_{G} x^{*}\right]_{0}\right)\right)^{\vee}, \text { by Lemma } 3.0 \\
& =\operatorname{Hom}_{A}\left(H_{m}^{1}\left(\left(0:_{A} x\right)\right), E_{A}(k)\right), \text { since }\left[0:_{G} x^{*}\right]_{0} \cong\left(0:_{A} x\right) \\
& \cong \operatorname{Hom}_{A}\left(\left(0:_{A} x\right), A\right), \text { by local duality and the fact that } w_{A} \cong A \\
& =\operatorname{Hom}(\operatorname{Hom}(A / I, A), A), \text { since }(0: x)=(0: I) \\
& \cong A / I, \text { since } A / I \text { is 1-dimensional Cohen-Macaulay, (cf. }[2, \text { Thm. 3.6]). }
\end{aligned}
$$

Hence sequence (6) in degree 0 is a split-sequence of $A / I$-modules. We conclude that

$$
\left[w_{G}\right]_{0} \cong\left[H_{M}^{1}\left(x^{*} G\right)^{\vee}\right]_{-1} \oplus A / I .
$$

Now we determine $\left[H_{M}^{1}\left(x^{*} G\right)^{\vee}\right]_{-1}$ as follows:

$$
\begin{aligned}
{\left[H_{M}^{1}\left(x^{*} G\right)^{\vee}\right]_{-1} } & \cong\left[H_{M}^{0}\left(G / x^{*} G\right)^{\vee}\right]_{-1}, \text { by }(7)\left(\text { since }\left[w_{G}\right]_{-1}=0\right) \\
& =\left(\left[H_{M}^{0}\left(G / x^{*} G\right)\right]_{1}\right)^{\vee}=\operatorname{Hom}_{A}\left(\left[H_{M}^{0}\left(G / x^{*} G\right)\right]_{1}, E_{A}\right) \\
& =\operatorname{Hom}_{A}\left(H_{m}^{0}(I / x A), E_{A}\right),(\text { by Lemma 3.0) }
\end{aligned}
$$


which by local duality is isomorphic to $\operatorname{Ext}_{A}^{1}(I / x A, A)$.

Finally we show that $\operatorname{Ext}_{A}^{1}(I / x A, A)=I / x A$ (together with (9), this proves the claim). Note that $\operatorname{Ext}_{A}^{1}(I / x A, A) \cong \operatorname{Ext}_{A}^{1}(A / x A, A)$, since $\operatorname{Ext}_{A}^{2}(A / I, A)=0$ (because $A$ is 1 dimensional Gorenstein) and $\operatorname{Ext}_{A}^{1}(A / I, A)=\operatorname{Hom}_{A}\left(H_{m}^{0}(A / I), E_{A}\right)=0$ (because $A / I$ is 1-dimensional Cohen-Macaulay). Now the sequence

$$
0 \rightarrow A /(0: I)=A /(0: x) \stackrel{x}{\rightarrow} A \rightarrow A / x A \rightarrow 0
$$

induces the exact sequence

$$
A=\operatorname{Hom}_{A}(A, A) \stackrel{x}{\rightarrow} \operatorname{Hom}_{A}(A /(0: I), A) \rightarrow \operatorname{Ext}_{A}^{1}(A / x A, A) \rightarrow 0
$$

where $\operatorname{Hom}_{A}(A /(0: I), A) \cong 0:(0: I)=I$, since $A / I$ is Cohen-Macaulay. Thus by $(10)$,

$$
\operatorname{Ext}_{A}^{1}(A / x A, A) \cong I / x A .
$$

3.2. Proof of Theorem 2.3. As in the proof of Theorem 2.1(ii) we will frequently apply Lemma 3.0, without mentioning it permanently. Furthermore, we have to use the following description of a minimal reduction of $l$, given by [11], Proposition 3.3 and its proof ].

Lemma 3.3. Under the assumptions of Theorem 2.3, a minimal reduction $J$ of $I$ can be generated by elements $a_{1}, \ldots, a_{h}, c, d$ satisfying the following properties:

(i) $a_{1}, \ldots, a_{h}$ is an $A$-regular sequence $\left(\mathbf{a}:=a_{1}, \ldots, a_{h}\right.$, for short);

(ii) $I_{P}=J_{P}=\mathbf{a}_{P}$ for $P \in \min _{A}(A / I)$;

(iii) $I_{q}=J_{q}=(\mathrm{a}, c) A_{q}$ for $q \supseteq I$, ht $(q / I)=1$; more precisely:

$$
I_{q}=\left(a_{1}, \ldots, a_{h-1}, c\right) A_{q} \text { if } q \supseteq I+\left(\mathbf{a}:_{A} I\right), \quad \operatorname{ht}(q / I)=1
$$

and

$$
I_{q}=\mathbf{a} A_{q} \text { if } q \nsupseteq\left(\mathbf{a}:_{A} I\right), \quad \operatorname{ht}(q / I)=1 ;
$$

(iv) $(\mathbf{a}: c)=(\mathbf{a}: I)$

(v) $(\mathbf{a}: c) \cap I=\mathbf{a}$

(vi) $((\mathbf{a}, c): d) \cap I=(\mathbf{a}, c)$.

If moreover $r(I) \leq 1$, then

(vii) $(\mathrm{a}, c) \cap l^{n}=(\mathrm{a}, c) I^{n-1}$ for $n \geq 1$;

(viii) $\mathrm{a} \cap I^{n}=\mathrm{a} I^{n-1}$ for $n \geq 1$; equivalently, the sequence $a_{1}^{*}, \ldots, a_{h}^{*}$ of initial forms forms a regular sequence on $G(I)$.

Remark. (1) The proof of Prop. 3.3 in [11] shows that properties (iv)-(viii) are consequences of the first three properties. Furthermore, property (vi) follows from the 
"first part" of (iii), by a result of Kustin, Miller and Ulrich. This was pointed out in the proof of Prop. 3.3 in [11].

(2) In case $r(I) \leq 1$ we know by (viii) that $a_{1}^{*}, \ldots, a_{h}^{*}$ form a regular sequence on $G(I)$. In case $r(I) \geq 2$ this is not clear $a$ priori. But in Lemma 3.4 below we shall construct a system of parameters for $G(I)$, with $a_{1}^{*}, \ldots, a_{h}^{*}$ being part of it. If $G(I)$ is $C M$, we have property (viii) also in the case $r(I) \geq 2$. This knowledge allows us to reduce questions to the case $h t(l)=0$.

Lemma 3.4. There is a s.o.p. of $G(I)$ of the type

$$
a_{1}^{*}, \ldots, a_{h}^{*}, c^{*}+x^{*}, d^{*}+y^{*}, z_{1}^{*}, \ldots, z_{d-h-2}^{*}
$$

where $x \in\left(\mathbf{a}:{ }_{A} c\right)$ and $y \in(\mathbf{a}, c):_{A} I$ are chosen such that $\mathbf{a}, c+x, d+y$ is a regular sequence on $A$ and such that $c+x, d+y$ is a $A / I$ regular sequence; moreover $z_{1}, \ldots, z_{d-h-2}, c, y+d$ is a s.o.p. of $A / I$. Furthermore a,,$d$ are a reduction of $I$ as in Lemma 3.3.

Proof of Lemma 3.4. We first note that this s.o.p. is more precisely an s.o.p. for $G_{M}$. However, we later will show that $M$ is the only maximal ideal containing these elements.

Using properties (ii) and (iii) in Lemma 3.3 together with McAdam's prime avoidance theorem, it is easy to see that

$$
\left(\mathbf{a}:{ }_{A} c\right) \nsubseteq \bigcup_{P \in A S S A(A / \mathbf{a})}-c+P
$$

hence there is $x \in\left(\mathbf{a}:_{A} c\right)$ such that $x+c$ is regular on $A / \mathbf{a}$. Now $\operatorname{Ass}_{A}(A / \mathbf{a}) \supseteq \operatorname{Ass}_{A}(A / I)$, because $A / I$ is $C M$; thus $x+c$ is also regular on $A / I$. Similarly one shows that

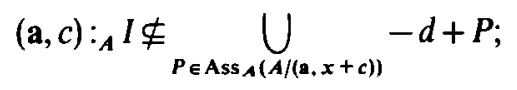

and finds $y \in(\mathbf{a}, c):_{A} I$ such that $y+d$ is regular on $A /(\mathbf{a}, x+c)$ and hence on $A /(I, x+c)$, too. More precisely, if

$$
(\mathrm{a}, c):{ }_{A} I \subseteq \bigcup_{P \in \operatorname{AsS}_{A}(A /(\mathbf{a}, x+c))}-d+P
$$

then by McAdam's prime avoidance lemma [14, Theorem 5], there is a prime $P$ associated with $A /(\mathrm{a}, x+c)$ such that $(\mathrm{a}, c):_{A} I+(d) \subseteq P$. Note that height $(P)=h+1$, since $x+c$ is regular on $A / \mathbf{a})$. Of course, $(\mathbf{a}, c, d)=J \subseteq P$, and hence $l \subseteq P$. This implies that $P A_{P}$ contains $\left((\mathrm{a}, c):_{A} I\right)_{P}=I_{P}: I_{P}=A_{P}$ which is a contradiction.

Now, let $x+c, y+d, z_{1}, \ldots, z_{d-n-2}$ be a s.o.p. of $A / I$.

We need to prove that $a_{1}^{*}, \ldots, a_{h}^{*}, c^{*}+x^{*}, d^{*}+y^{*}, z_{1}^{*}, \ldots, z_{d-h-2}^{*}$ is a s.o.p. for $G(I)$. It suffices to prove that the ideal $G_{+}$of positive degree elements of $G=G(I)$ is in the ideal 
$Q$ generated by these elements up to radical, since $G / G_{+} \cong A / I$, and the images of these elements are a s.o.p. for $A / I$. Up to radical $G_{+}$is generated by the images of the reduction of $I, a_{1}^{*}, \ldots, a_{h}^{*}, c^{*}, d^{*}$, and it therefore is enough to prove that both $c^{*}$ and $d^{*}$ are in the nilradical of $Q$. But the choice of $x$ and $y$ prove that $\left(c^{*}\right)^{2}=c^{*}\left(c^{*}+x^{*}\right)$ modulo $\left(a_{1}^{*}, \ldots, a_{h}^{*}\right)$, and $\left(d^{*}\right)^{2}=d^{*}\left(d^{*}+y^{*}\right)$ modulo $a_{1}^{*}, \ldots, a_{h}^{*}, c^{*}$.

The next two results show that we may reduce to the case $h=0, d=2$ without changing the properties mentioned in Lemma 3.4, provided that $G(I)$ is Cohen-Macaulay.

Lemma 3.5. Assume that $G(I)$ is Cohen-Macaulay. Let a, $, d, x, y$ be as in Lemma 3.4. Set $\bar{A}:=A / \mathbf{a}, \bar{I}:=I / \mathbf{a}, \bar{J}:=J / \mathbf{a}, G:=\bar{G}(\bar{I})$. Then

(a) $\bar{J}$ is a min. reduction of $\bar{I}$ and $r_{\bar{J}}(\bar{I})=r_{J}(I) ; \bar{G}=G(I) / \mathrm{a}^{*}$ is $C M$ and $a(\bar{G})=a(G(I))+h$;

(b) the corresponding properties (ii), (iii), (iv), (v), (vi) in Lemma 3.3 hold for $\bar{I}$ in place of $I$, and if $r(I) \leq 1$ then (vii) in Lemma 3.3 holds for $I$ instead of $I$.

Proof. (a) is well-known and (b) is easy to see.

Lemma 3.6. Adopt the notation and assumptions of Lemma 3.5. Let I be the ideal $\bar{I}$ of Lemma $3.5($ i.e. $\mathrm{ht}(I)=0)$. If $d \geq 3(d=\operatorname{dim} A)$, there is an element $u \in m \backslash I$, such that $u^{*}$ is regular on $G(I)$ and which satisfies the following properties (where $\bar{A}:=A / u A, \bar{I}:=(I, u) /$ $(u), \bar{J}=(J, u) /(u), \bar{G}:=\bar{G}(\bar{I}))$ :

(a) $\operatorname{ht}(\bar{I})=0, \operatorname{dim}(\bar{A})=d-1, a(\bar{G})=a(G(I))$;

(b) $\bar{J}$ is a minimal reduction of $\bar{I}$ and $r_{\bar{J}}(\bar{I})=r_{J}(I)$;

(c) $\bar{I}_{\bar{P}}=\overline{0}$ for $\bar{P} \in \min _{\bar{A}}(\bar{A} / \bar{I})$;

(d) $\bar{I}_{\bar{Q}}=\bar{c} \bar{A}_{\bar{Q}}$ for $\bar{Q} \supseteq \bar{I}$, ht $(\bar{Q} / \bar{I})=1$.

Proof. Consider the sets

$$
B:=\left\{P \in \operatorname{Spec}(A) \mid \mathrm{ht}(P)=1, I \subseteq P, I_{P} \neq 0\right\}
$$

and

$$
C:=\left\{Q \in \operatorname{Spec}(A) \mid \mathrm{ht}(Q)=2, I \subseteq Q, I_{Q} \neq c A_{Q}\right\}
$$

Since $B \subseteq \min _{A} A /\left(I+\left(0:_{A} I\right)\right)$ and $C \subseteq \min _{A}\left(A /\left(I+a n n_{A}(I / c A)\right), B\right.$ and $C$ are both finite sets. Now

$$
m /\left(m^{2}+I\right) \supsetneq \bigcup_{P \in \operatorname{Ass} G(I)} \frac{P \cap A+I+m^{2}}{I+m^{2}} \cup \bigcup_{P \in B} \frac{P+m^{2}+I}{m^{2}+I} \bigcup_{Q \in C} \frac{Q+m^{2}+I}{m^{2}+I}
$$

(otherwise $(m / I) \subseteq P$ for some $P \in \operatorname{Ass}(G(I))$ (note that $m \notin B \cup C$, since ht $(m)=d \geq 3$ ) and hence grade $(m G(I))=0$; but on the other hand $h t(m G(I))=d-\ell(I) \geq 1$ (since $d \geq 3$ and $\ell=h+2=2$ ), a contradiction). 
Hence we may choose $u \in m \backslash I$, such that $u^{*}$ is regular on $G(I)$ and $u \notin \bigcup_{P \in B} P$, $u \notin \bigcup_{Q_{E} C} Q$. Properties (a) and (b) are already well-known (and easy to check). We demonstrate here only (d). Part (c) can be done similarly. Note that a prime $\bar{Q} \supseteq \bar{I}$, $1=\mathrm{ht}(\bar{Q} / \bar{I})$ corresponds to a prime $Q \supseteq(I, u)$, ht $(Q)=2$ and that

$$
\frac{\bar{I}_{\bar{Q}}}{\bar{c} A_{\bar{Q}}}=\frac{I_{Q}+u A_{Q}}{c A_{Q}+u A_{Q}} \quad\left(\text { where } \bar{Q}=\frac{Q}{u A}\right)
$$

since $u \in Q$, we have $Q \notin C$ (by choice of $u$ ), hence $I_{Q}=c A_{Q}$, so that also in this case the fraction is zero. This establishes property (d).

Remark 3.7. Using Lemma 3.5 and Lemma 3.6 we restrict from now on to the situation $h=0, d=2$, in which we shall prove the assertions of Theorem 2.3. For convenience we describe this situation here: $h t(I)=0, \quad \operatorname{dim}(A)=2, \quad I_{P}=0$ for $P \in \min _{A}(A / I), J=(c, d)$ is a minimal reduction of $I, I_{Q}=c A_{Q}$ if $I \subseteq Q$ and ht $(Q)=1, A$ is Gorenstein and $A / I$ Cohen-Macaulay; moreover the following relations hold $(0 ; I)=(0: c),(0: I) \cap I=(0),((c): d) \cap I=(c)$. In case $r(I) \leq 1$, we know moreover that (c) $\cap I^{n}=c I^{n-1}$ for all $n \geq 1$. Moreover $x I=0$, and $y I \subseteq(c)$.

For the computation of the $a$-invariant as announced in Theorem 2.3 we need the following Lemma 3.8 .

Lemma 3.8. Let the situation $(h=0, d=2$, etc.) be as described in Remark 3.7. Further assume that $G(I)$ is Cohen-Macaulay. Then:

(a) $\left[\left(0: G c^{*}\right)\right]_{n}=0$ for $n \geq 1$;

(b) $\left.\left[\left(c^{*}\right):_{G} d^{*}\right) /\left(c^{*}\right)\right]_{n}=0$ for $n \geq 2$. If $r(I) \leq 2$, then this equation holds for $n=1$.

Proof of (a). By Lemma 3.4, $c^{*}+x^{*}, d^{*}+y^{*}$ form an s.o.p. for $G$. Since $G$ is Cohen-Macaulay, these two elements form a regular sequence. Let $\alpha \in I^{n}, c \alpha \in I^{n+2}$. Here $n \geq 1$. Since $x I=0$, we then obtain that $\alpha^{*}\left(c^{*}+x^{*}\right)=0$. It follows that $\alpha^{*}=0$, which proves (a).

Proof of (b). Fix $n \geq 2$, and let $u^{*}$ represent an element in $\left.\left[\left(c^{*}\right):_{G} d^{*}\right) /\left(c^{*}\right)\right]_{n}$. Then there exists an element $v^{*} \in G_{n}$ such that $u^{*} d^{*}+v^{*} c^{*}=0$. Since $y I \subseteq(c)$, we know that $y u \in y I^{n}=(y I)\left(I^{n-1}\right) \subseteq c I^{n-1}$. Hence we can write $u^{*}\left(d^{*}+y^{*}\right)+\left(w^{*}+v^{*}\right) c^{*}=0$ for some $w^{*} \in G_{n-1}$. Since $n \geq 2$, it follows that $x^{*}\left(w^{*}+v^{*}\right)=0$, so that $u^{*}\left(d^{*}+y^{*}\right)+\left(w^{*}+v^{*}\right)\left(c^{*}+x^{*}\right)=0$. Since $c^{*}+x^{*}, d^{*}+y^{*}$ form a regular sequence, we obtain that $u^{*} \in\left(c^{*}+x^{*}\right) G$. As $x^{*}$ kills all positive degree elements of $G$ it follows that $u^{*} \in c^{*} G$, as required.

Now assume that $r(I) \leq 2$. Let $u^{*}$ be as above except assume it has degree 1 . Lift $u^{*}$ and $v^{*}$ to $A$, where $v^{*}$ is as in the above paragraph. Then there is an equation $u d+v c \in I^{3}=(c, d) I^{2}$. Changing $u$ by an element of $I^{2}$ (which doesn't change $u^{*}$ ), we see that $u \in((c): d) \cap I=(c)$. Hence $u^{*} \in c^{*} G$, as needed. 
Proof of Theorem 2.3. Now we compute that the $a$ invariant $a(G(I))=r-2$ in case $r \geq 2$ (the case $r \leq 1$ being similar), $h=0, d=2$. We work with the following four exact sequences of graded $G$-modules, where $G:=G(I)$.

$$
\begin{aligned}
& 0 \rightarrow\left(0:_{G} c^{*}\right) \rightarrow G \stackrel{c^{*}}{\rightarrow}\left(c^{*} G\right)(1) \rightarrow 0 \\
& 0 \rightarrow c^{*} G \rightarrow G \rightarrow G / c^{*} G \rightarrow 0 \\
& 0 \rightarrow\left(c^{*}:_{G} d^{*}\right) /\left(c^{*}\right) \rightarrow G /\left(c^{*}\right) \stackrel{d^{*}}{\rightarrow}\left(\left(c^{*} d^{*}\right) /\left(c^{*}\right)\right)(1) \rightarrow 0 \\
& 0 \rightarrow\left(c^{*}, d^{*}\right) /\left(c^{*}\right) \rightarrow G /\left(c^{*}\right) \rightarrow G /\left(c^{*}, d^{*}\right) \rightarrow 0 .
\end{aligned}
$$

Consider the induced long exact cohomology sequences $\left(H^{i}(-):=H_{M}^{i}(-)\right)$

(1) $0 \rightarrow H^{1}\left(c^{*} G\right)(1) \rightarrow H^{2}\left(0:_{G} c^{*}\right) \rightarrow H^{2}(G) \rightarrow H^{2}\left(c^{*} G\right)(1) \rightarrow 0$

(2) $H^{0}\left(G / c^{*} G\right) \cong H^{1}\left(c^{*} G\right)$

$0 \rightarrow H^{1}\left(G / c^{*} G\right) \rightarrow H^{2}\left(c^{*} G\right) \rightarrow H^{2}(G) \rightarrow H^{2}\left(G / c^{*} G\right) \rightarrow 0$

(3) $\ldots \rightarrow H^{i}\left(\left(c^{*}: d^{*}\right) /\left(c^{*}\right)\right) \rightarrow H^{i}\left(G /\left(c^{*}\right)\right) \rightarrow H^{i}\left(\left(c^{*}, d^{*}\right) /\left(c^{*}\right)\right)(1) \rightarrow$ $H^{i+1}\left(\left(c^{*}: d^{*}\right) /\left(c^{*}\right)\right) \rightarrow \cdots$

(4) $\ldots \rightarrow H^{i-1}\left(G /\left(c^{*}, d^{*}\right)\right) \rightarrow H^{i}\left(\left(c^{*}, d^{*}\right) /\left(c^{*}\right)\right) \rightarrow H^{i}\left(G /\left(c^{*}\right)\right) \rightarrow H^{i}\left(G /\left(c^{*}, d^{*}\right)\right) \rightarrow$ $H^{i+1}\left(\left(c^{*}, d^{*}\right) /\left(c^{*}\right)\right) \rightarrow \cdots$

Note that in (3) $\left[H^{i}\left(\left(c^{*}, d^{*}\right) /\left(c^{*}\right)\right)\right]_{n}=0=\left[H^{i+1}\left(\left(c^{*}, d^{*}\right) /\left(c^{*}\right)\right)\right]_{n}$ for $n \geq 2$ by Lemma 3.8, while in (4) $\left[H^{i-1}\left(G /\left(c^{*}, d^{*}\right)\right)\right]_{n}=0=\left[H^{i}\left(G /\left(c^{*}, d^{*}\right)\right)\right]_{n}=0$ for $n \geq r+1$. Thus we have isomorphisms

(3) $\left[H^{i}\left(G /\left(c^{*}\right)\right)\right]_{n} \cong\left[H^{i}\left(\left(c^{*}, d^{*}\right) /\left(c^{*}\right)\right)\right]_{n+1}$ for $n \geq 2$, and

$(4)^{\prime}\left[H^{i}\left(\left(c^{*}, d^{*}\right) /\left(c^{*}\right)\right)\right]_{n} \cong\left[H^{i}\left(G /\left(c^{*}\right)\right)\right]_{n}$ for $n \geq r+1$.

Since $\left[H^{i}\left(G /\left(c^{*}\right)\right)\right]_{n}=0$ for all $n \gg 0$, we conclude that $\left[H^{i}\left(G /\left(c^{*}\right)\right)\right]_{n}=0$ for $n \geq r$. Moreover, in (1) we have $\left[H^{2}\left(\left(0:_{G} c^{*}\right)\right)\right]_{n}=0$ for $n \geq 1$ (by Lemma 3.8) and in (2) $\left[H^{1}\left(G /\left(c^{*}\right)\right)\right]_{n}=0=\left[H^{2}\left(G / c^{*} G\right)\right]_{n}$ for $n \geq r$ (as we have just shown). Hence the sequences (1) and (2) give isomorphisms

(5) $\left[H^{2}(G)\right]_{n} \cong\left[H^{2}\left(c^{*} G\right)\right]_{n+1}$ for $n \geq 1$

(6) $\left[H^{2}\left(c^{*} G\right)\right]_{n} \cong\left[H^{2}(G)\right]_{n}$ for $n \geq r$.

As $\left[H^{2}(G)\right]_{n}=0$ for all $n \gg 0$, we conclude that $\left[H^{2}(G)\right]_{n}=0$ for $n \geq r-1$. This means $a(G(I)) \leq r-2$. It remains to show that $\left[H^{2}(G)\right]_{r-2} \neq 0$. For that, note that in (4) $\left[H^{0}\left(G / c^{*} G\right)\right]_{r}=0$ and $\left[H^{0}\left(G /\left(c^{*}, d^{*}\right)\right)\right]_{r} \neq 0$ (because $\left[H^{0}\left(G /\left(c^{*}, d^{*}\right)\right)\right]_{r}=H_{m}^{0}\left(I^{r} /(c, d) I^{r-1}\right)=$ $\left.I^{r} /(c, d) I^{r-1} \neq 0\right)$; thus $\left[H^{1}\left(\left(c^{*}, d^{*}\right) /\left(c^{*}\right)\right)\right]_{r} \neq 0$ by $(4)$. Now $(3)^{\prime}$ with $i=1$ gives $\left[H^{1}\left(G /\left(c^{*}\right)\right)\right]_{r-1} \neq 0$. Hence in $(2)\left[H^{2}\left(c^{*} G\right)\right]_{r-1} \neq 0$ and since, by $(1),\left[H^{2}\left(c^{*} G\right)\right]_{r-1} \neq 0$ is 


\section{REDUCTION EXPONENTS OF IDEALS WITH GORENSTEIN FORMRING 461}

a homomorphic image of $\left[H^{2}(G)\right]_{r-2}$, we conclude that $\left[H^{2}(G)\right]_{r-2} \neq 0$, proving $a(G)=r-2$.

Since the proof of the formula $a(G)=0$ in case $r \leq 1$ uses similar arguments, we omit it here. Instead, we prove now the second part of Theorem 2.3, saying that $G$ cannot be Gorenstein if $r \geq 2$. Denote by $w_{G}$ the canonical module of $G$, i.e. $w_{G}=$ $\operatorname{Hom}_{G}\left(H^{2}(G), E_{G}(k)\right)$. Since $a(G)=r-2$, it is enough to show that

$$
\left[w_{G}\right]_{-r+2} \not A / I\left(=G(r-2)_{-r+2}\right) \text {. }
$$

For that, apply the functor $\operatorname{Hom}_{G}\left(-, E_{G}(k)\right) \equiv \operatorname{Hom}_{A}\left(-, E_{A}(k)\right)$ to the above sequences (1), (2), (3), (4); the new sequences are:

$(1)^{\vee} 0 \rightarrow H^{2}\left(c^{*} G\right)^{\vee}(-1) \rightarrow w_{G} \rightarrow H^{2}\left(0:_{G} c^{*}\right)^{\vee} \rightarrow H^{1}\left(c^{*} G\right)^{\vee}(-1) \rightarrow 0$

(2) ${ }^{\vee} H^{1}\left(c^{*} G\right)^{\vee} \cong H^{0}\left(G / c^{*} G\right)^{\vee}$,

$$
0 \rightarrow H^{2}\left(G / c^{*} G\right)^{\vee} \rightarrow w_{G} \rightarrow H^{2}\left(c^{*} G\right)^{\vee} \rightarrow H^{1}\left(G / c^{*} G\right)^{\vee} \rightarrow 0
$$

$(3)^{\vee} H^{i+1}\left(\left(c^{*}: d^{*}\right) /\left(c^{*}\right)\right)^{\vee} \rightarrow H^{i}\left(\left(c^{*}, d^{*}\right) /\left(c^{*}\right)\right)^{\vee}(-1) \rightarrow H^{i}\left(G / c^{*} G\right)^{\vee} \rightarrow$ $H^{i}\left(\left(c^{*}: d^{*}\right) /\left(c^{*}\right)\right)^{v}$

$(4)^{\vee} H^{i}\left(G /\left(c^{*}, d^{*}\right)\right)^{\vee} \rightarrow H^{i}\left(G / c^{*} G\right)^{\vee} \rightarrow H^{i}\left(\left(c^{*}, d^{*}\right) /\left(c^{*}\right)\right)^{\vee} \rightarrow H^{i-1}\left(G /\left(c^{*}, d^{*}\right)\right)^{\vee} \rightarrow$ $H^{i-1}\left(G /\left(c^{*}\right)\right)^{v}$.

Consider the first module in $(1)^{\vee}$ in degree $-r+2$ :

$$
\begin{aligned}
& {\left[H^{2}\left(c^{*} G\right)^{\vee}(-1)\right]_{-r+2}=\left[H^{2}\left(c^{*} G\right)^{\vee}\right]_{-r+1}} \\
& \cong\left[H^{1}\left(G / c^{*} G\right)^{\vee}\right]_{-r+1}, \text { by }(2)^{\vee}\left(\text { use }\left[w_{G}\right]_{-r+1}=0, \text { since } a(G)=r-2\right) \\
& \cong\left[H^{1}\left(\left(c^{*}, d^{*}\right) /\left(c^{*}\right)\right)^{\vee}\right]_{-r}, \text { by }(3)^{\vee}\left(\text { use }\left[\left(c^{*}: d^{*}\right) /\left(c^{*}\right)\right]_{r-1}=0\right) \\
& \left.\cong\left[H^{0}\left(G /\left(c^{*}, d^{*}\right)\right)^{\vee}\right]_{-r}, \text { by }(4)^{\vee} \text { (use }\left[H^{i}\left(G /\left(c^{*}\right)\right)\right]_{r}=0\right) \\
& \quad=H_{m}^{0}\left(I^{r} /(c, d) I^{r-1}\right)^{\vee},
\end{aligned}
$$

which is a module $\neq 0$ of finite length over $A$ (since $I_{q}=c A_{q}$ for all primes $q$, such that $I \subseteq q$, ht $(q) \leq 1)$.

We have shown that $\left[w_{G}\right]_{-r+2}$, in sequence $(1)^{v}$, contains a non-zero submodule of finite length; hence $H_{m}^{0}\left(\left[w_{G}\right]_{-r+2}\right) \neq 0$ and we conclude $\left[w_{G}\right]_{-r+2} ¥ A / I$. That finishes the proof of Theorem 2.3 .

Proof of Theorem 2.5. First observe that $k$ exists, because $I^{j}: m \subseteq I^{j}: I=I^{j-1}$ as $G$ is Cohen-Macaulay, and $I^{j-1} \subseteq J$ for $j \gg 0$. Let $u \in I^{k}: m \backslash\left(I^{k}+J\right)$. Then $u \in I^{k-1} \backslash I^{k}$.

Claim. $\overline{u^{*}}$ is in the socle of $\bar{G}:=G /\left(x_{1}^{*}, \ldots, x_{d}^{*}\right)$. 
Proof of the claim. : If $y \in I \backslash I^{2}$, then

$$
y \cdot u \in I\left(I^{k}: m\right) \subseteq I^{k+1}: m \subseteq I^{k+1}+J,
$$

the last containment by choice of $k$. This implies

$$
y \cdot u \in I^{k+1}+I^{k} \cap J=I^{k+1}+J I^{k-1}
$$

as $G$ is Cohen-Macaulay. Hence $\bar{y}^{*} \cdot \bar{u}^{*}=0$. If $z \in m \backslash I$, then $z u \in I^{k}$ by definition of $u$, and so also $\overline{z^{*}} \cdot \overline{u^{*}}=0$, which proves the claim.

Hence $\bar{G}=G /\left(x_{1}^{*}, \ldots, x_{d}^{*}\right)$ has a non-zero socle element in degree $k-1$, i.e. $[\bar{G}]_{n}=0$ if $n \geqq k$ since $\bar{G}$ is Gorenstein. Thus $I^{k}=I^{k+1}+J I^{k-1}$ which implies that $I^{k}=J \cdot I^{k-1}$, i.e. $r_{J}(I) \leqq k-1$. Therefore $r_{J}(I)=k-1$ since $I^{k-1} \not J$ (else $\left.I^{k}: m \subseteq J\right)$.

Now assume that $A$ is regular. It remains to see that $k \leqq d-1$. For this we use a result of Lipman [13], saying that for any $m$-primary ideal in a regular local ring $(A, m)$ with minimal reduction $J$ we have

$$
I^{d}: m^{d-1} \subseteq J .
$$

Hence $I^{d}: m \subseteq I^{d}: m^{d-1} \subseteq J$ which proves that $k \leqq d-1$.

Proof of Corollary 2.6. The "only if" part is obvious. On the other hand, if $A / I$ is Gorenstein then $I$ is a complete intersection by [15, Theorem 2.6] since $I$ is a perfect ideal, so $r(I)=k-1=0$.

Proof of Corollary 2.7. (i) implies $r(I) \leqq d-2$ by Theorem 2.5. Hence we get for the $a$-invariant of $G$ :

$$
a:=a(G)=r(I)-d \leqq-2, \quad \text { see }[9,(2.4)] .
$$

Then $q:=-a-1 \geqq 1$ is the desired exponent of $I$ such that $R\left(I^{q}\right)$ is Gorenstein by [9, Theorem 3.5], which proves (ii). We also note that the remark following Corollary 2.6 shows that $R(I)$ is Cohen-Macaulay.

Conversely assuming (ii), $G(I)$ must be Gorenstein by [8, Theorem 2.3], because $G(I)$ is Cohen-Macaulay (by the general assumption) which implies also the CohenMacaulayness of $R(I)$ in the situation (ii).

\section{REFERENCES}

1. I. M. Aberbach and C. Huneke, An improved Briançon-Skoda theorem with applications to the Cohen-Macaulayness of Rees algebras, Math. Ann. 297 (1993), 343-369.

2. E. G. Evans and P. Grifith, Syzygies (London Math. Soc. Lecture Note Senes 103, Cambridge University Press, Cambridge, 1985).

3. S. Goto and S. Huckaba, On graded rings associated to analytic deviation one ideals, Amer. J. Math. 116 (1994), 905-919. 


\section{REDUCTION EXPONENTS OF IDEALS WITH GORENSTEIN FORMRING 463}

4. S. Goto and Y. Nakamura, On the Gorensteinness of graded rings associated to ideals of analytic deviation one, (1992), preprint.

5. S. Goto and Y. NaKamURA, Gorenstein graded rings associated to ideals of analytic deviation two, (1993), preprint.

6. M. Herrmann, S. Ikeda and U. Orbanz, Equimultiplicity and Blowing Up (Springer-Verlag, Berlin-Heidelberg, 1988).

7. J. Herzog, A. Simis and W. V. VAsconcelos, Koszul homology and blowing up rings (Lecture Notes in Pure and Appl. Math. 84, Marcel Dekker, New York 1983), 79-169.

8. M. Hermmann, J. Ribbe and P. Schenzel, On the Gorenstein property of form rings, Math. Z. 213 (1993), 301-309.

9. M. Hermmann, J. Ribbe and S. Zarzuela, On the Gorenstein property of Rees and form rings of powers of ideals, Trans. Amer. Math. Soc., to appear.

10. S. Huckaba and C. Huneke, Powers of ideal having small analytic deviation, Amer. J. Math. 114 (1992), 367-403.

11. S. Huckaba and C. Huneke, Rees algebras of ideals having small analytic deviation, Trans. Amer. Math. Soc. 339 (1993), 373-402.

12. C. Huneke, Linkage and the Koszul homology of ideals, Amer. J. Math. 104 (1982), 1043-1062.

13. J. Lipman, Adjoint of ideals in regular local rings, Math. Res. Letters 1 (1994), 1-17.

14. S. McAdam, Finite coverings of ideals, in Ring Theory (Proc. of the Oklahoma Conference, Lecture Notes in Pure and Appl. Math., Marcel Dekker 7 1974), 163-171.

15. S. Noh and W. V. Vasconcelos, The $S_{2}$-closure of a Rees algebra, Results in Math. 23 (1993), 149-162.

16. K. N. RaGhavan, Uniform annihilation of local cohomology and powers of ideals generated by quadratic sequences (Thesis, Purdue 1991).

Mathematisches Institut der Univ. zu Koeln

WeYERTAL 86-90

D-50931 KOELN

Germany

E-mail address: herrmann@mi.uni-koeln.de

Mathematisches Institut der Univ. zU Koeln

WEYERTAL 86-90

D-50931 KOELN

Germany

E-mail address: ribbe@mi.uni-koeln.de
Department of Mathematics

Purdue University

WEst LAFAyETTE, IN

USA

E-mail address: huneke@math.purdue.edu 